\title{
émulations
}

\section{Anne Gillet, Diane-Gabrielle Tremblay (dir.) - Les recherches partenariales et collaboratives}

Jeanne Demoulin

Émulations - Revue de sciences sociales

2019. Comptes rendus critiques. En ligne

\section{Article disponible à l'adresse suivante}

https://ojs.uclouvain.be/index.php/emulations/article/view/7493

\section{Pour citer cet article}

Jeanne Demoulin, « Anne Gillet, Diane-Gabrielle Tremblay (dir.) - Les recherches partenariales et collaboratives », Émulations, en ligne. Mise en ligne le 12 mars 2019. DOI : 10.14428/emulations.cr.048

Distribution électronique : Université catholique de Louvain (Belgique) : ojs.uclouvain.be

(C) Cet article est mis à disposition selon les termes de la Licence Creative Commons Attribution, Pas d'Utilisation Commerciale 4.0 International. http://creativecommons.org/licenses/by$\mathrm{nc} / 4.0 /$

Éditeur : Émulations - Revue de sciences sociales / Presses universitaires de Louvain https://ojs.uclouvain.be/index.php/emulations

ISSN électronique : 1784-5734

UCL PRESSES

UNIVERSITAIRES 


\title{
Anne Gillet, Diane-Gabrielle Tremblay (dir.) - Les recherches partenariales et collaboratives
}

\begin{abstract}
Jeanne Demoulin ${ }^{1}$
Recensé : Anne Gillet, Diane-Gabrielle Tremblay (dir.), Les recherches partenariales et collaboratives, Rennes/Québec, Presses universitaires de Rennes/Presses de l'Université du Québec, 2017.
\end{abstract}

Les recherches collaboratives et partenariales ont pour principe d'associer des chercheurs professionnels et des chercheurs non professionnels. Elles semblent connaître un regain d'intérêt ces dernières années au sein de la communauté scientifique internationale. Nombre d'auteurs ont ainsi noté que la recherche serait de moins en moins la prérogative des seuls universitaires (René, Champagne, Margau, 2013) dans un contexte où les demandes de participation se multiplient, où les technosciences sont remises en question et où, bien entendu, les sources de financement pour la recherche sont plus difficiles à trouver : «Pour les scientifiques la recherche partenariale est aussi une manière d'obtenir de nouveaux contrats de recherche et/ou de légitimer des postures et des pratiques innovantes dans le champ académique» (Deroubaix, De Conink, 2013). Des professionnels du public comme du privé, des «habitants » ou usagers, mais aussi des élus se mettent ainsi «en recherche». Si ce type de recherche reste relativement marginal en France, il a été largement mis en pratique, institutionnalisé et théorisé dans d'autres régions, et notamment au Québec. C'est ce dont témoigne en outre le processus de production et le contenu même de l'ouvrage réalisé sous la direction d'Anne Gillet et de Diane-Gabrielle Tremblay. Les textes qui y sont réunis sont en effet le fruit des travaux menés depuis 2011 par un collectif de recherche composé de scientifiques français, québécois et suisses œuvrant dans le champ des sciences humaines et sociales et principalement en sociologie. À partir de leurs expériences de terrain et de leurs lectures en la matière, les auteurs proposent, en accord avec l'objectif de l'ouvrage, des apports théoriques et conceptuels approfondis pour analyser et comprendre les dynamiques et les enjeux des recherches partenariales et collaboratives. Des expériences conduites dans des contextes divers sont ainsi analysées, comme le programme francilien aujourd'hui disparu «Partenariat institutions-citoyens pour la recherche et l'innovation » qui promouvait des recherches entre universitaires et acteurs de la société civile (Christine Audoux), le réseau Développement durable et lien social (2DLiS)

${ }^{1}$ Post-doctorante en études urbaines, LAVUE-Mosaïques, UMR CNRS 7218. 
qui allie universités du Nord et du Sud et des acteurs locaux concernés par les problématiques du réseau (Maurice Blanc et Josiane Stoessel-Ritz) ou encore un «incubateur universitaire» au Québec, Parole d'excluEs, qui regroupe une pluralité d'acteurs pour «travailler collectivement avec et en fonction des besoins portés et exprimés par des personnes pauvres ou exclues» (p. 239, Jean-Marc Fontan).

Comme signalé dans l'introduction de l'ouvrage, ce livre permet non seulement de mettre en évidence la multiplicité de pratiques regroupées sous ces appellations, mais également leur diversité terminologique. Si le titre mentionne les recherches «partenariales » et "collaboratives», les auteurs utilisent en effet bien d'autres expressions pour qualifier et analyser les pratiques existantes, comme, par exemple, les «recherches partenariales participatives» (Yves Bonny), la "recherche participative» (Rémi Barré), la «recherche conjointe ( (Philippe Lyet) ou encore la «recherche-active » que les coordinatrices de l'ouvrage ajoutent en conclusion. On serait alors bien avisé de suivre le conseil d'Yves Bonny qui, réfléchissant aux typologies possibles dans le chapitre dont il est l'auteur, signale qu'il existe un «flottement considérable de la terminologie selon les auteurs et les courants » : il convient alors de "ne pas se focaliser sur les appellations, qui sont tout sauf contrôlées, mais sur les définitions et les distinctions conceptuelles et théoriques auxquelles elles sont associées» (p. 25).

L'ouvrage, riche, est composé de quatorze contributions, d'une introduction et d'une conclusion. Si l'introduction et la conclusion donnent des pistes pour lire ensemble les contributions et rassembler les enjeux structurants, la variété et le grand nombre de textes donnent une sensation d'éparpillement des problématiques abordées. Cela est renforcé par l'absence de parties dans l'ouvrage et la variété des vocables utilisés, comme mentionné plus haut. Pour autant, la structure de l'ouvrage et la profusion d'analyses ont le mérite de bien montrer que les pratiques sont loin d'être unifiées et que le champ reste largement en construction. Dans un souci de synthèse et de lisibilité, je ne reviendrai donc pas successivement sur chacune des contributions, mais je mettrai en évidence quelques éléments qu'il m’apparaît important de souligner ou qui m’ont interpelée.

Pour commencer, afin de bien saisir le cadre dans lequel se situe cet ouvrage, notons que les contributions s'intéressent davantage aux recherches menées avec des praticiens qu'à celles menées avec des citoyens ou usagers même si, comme cela est rappelé en introduction, il n'y a pas toujours de volonté d'action transformatrice dans les recherches analysées : la finalité peut être cognitive et épistémique. Assez classiquement, parce qu'il s'agit en effet d'un enjeu structurant, plusieurs contributions apportent des éléments sur la manière dont des acteurs aux statuts différents (salariés de structures privées ou publics, citoyens, usagers, universitaires...) peuvent parvenir à travailler ensemble. Diane-Gabrielle Tremblay et Juliette Rochman explorent par exemple «les processus de collaboration et de partage des connaissances » et plaident pour que chaque type d'acteur assume son identité et les tensions qui peuvent en émaner. Selon elles, 
c'est précisément la confrontation de ces différentes identités qui permet la construction de nouvelles connaissances. La contribution de Jacques Caillouette et Sid Ahmed Soussi va également dans ce sens en développant la notion d'«espace partenarial de recherche» dans laquelle les identités de chacun ne sont pas fusionnées, mais articulées et connectées les unes aux autres. Fontan revient pour sa part sur la difficulté de changer les postures traditionnelles, les professionnels considérant par exemple majoritairement que la théorie est réservée au monde de la recherche et se montrant intéressés par les résultats de la recherche, mais peu enclins à s'y impliquer. Comme cela a pu être montré ailleurs, les personnes que l'on souhaite impliquer en dehors des chercheurs peuvent se montrer très sceptiques et ne pas voir où se situe leur intérêt (Corwall et Jewkes, 1995).

La question de la légitimité scientifique est récurrente dans les analyses portant sur ce type de recherches. Les recherches partenariales se sont vues et se voient encore aujourd'hui accusées de se situer hors du champ de la recherche scientifique. Elles pâtissent souvent d'un manque de reconnaissance patent de la part de la communauté scientifique. Cette dimension est ici abordée par plusieurs contributions et particulièrement discutée par Lucie Dumais. L'auteure ne se fait pas l'avocate de toutes les formes de recherches partenariales : elle contribue au débat en définissant les règles qu'il convient, d'après elle, de respecter pour que la recherche soit effectivement valable ou puisse être reconnue scientifiquement. Le point névralgique se situe selon elle autour de la question de l'engagement du chercheur : «je distingue des degrés de politisation qui, au-delà de certains seuils, peuvent mettre en péril la légitimité même de la recherche sociale» (p. 63) expose-t-elle d'entrée de jeu. Elle critique vivement des démarches comme celle d'ATD Quart Monde, ou la posture défendue par Judith Butler, qui, comme aveuglées par un objectif politique de reconnaissance des acteurs sociaux, feraient fi d'acquis scientifiques ou les mettraient illégitimement en question: «ce genre de discours nous entraîne dans une logique négative - parfois dénigrante - de l'importance de la recherche, puisqu'il présuppose que la science n'a pu, par ses propres moyens [...] produire des connaissances suffisamment valables pour comprendre ce que vivent les populations marginalisées, ni pour reconnaître leurs savoirs d'expérience et leur dignité» (p. 75). Les recherches partenariales doivent ainsi, pour être légitimes, faire preuve d'une réelle rigueur scientifique et méthodologique. Si l'on peut être d'accord avec de tels points de vigilance, les débats autour de la «neutralité axiologique» ne sont pas nouveaux et l'on renverra pour notre part aux analyses qui montrent qu'un chercheur peut produire des recherches scientifiques tout en étant engagé dans le monde social qu'il analyse (Naudier, Simonet, 2011), dans les recherches partenariales comme dans d'autres formes de recherche.

Par ailleurs, il peut être important de ne pas «brouiller la frontière» entre différents types de savoirs (p. 75). Toutefois, la promulgation d'une hiérarchisation des savoirs dans laquelle le savoir scientifique est défini hors cadre comme supérieur à d'autres 
types de savoirs irait à contre-courant des velléités de nombreux membres de la communauté scientifique, mais également des systèmes institutionnels de recherche. Les chercheurs peuvent avoir été formés à penser que leur savoir est supérieur à d'autres (Cornwall, Jewkes, 1995), mais le texte de Barré, situé juste avant celui de Dumais dans l'ouvrage, montre précisément, comme d'autres avant lui (Callon, Lascoumes, Barthe, 2001), que la confrontation des savoirs est essentielle pour apporter de meilleures solutions aux problèmes rencontrés. Cette dimension est envisagée comme un point d'ancrage du «nouveau contrat science-société», dont les maîtres-mots sont participation et réflexivité. Barré appelle ainsi pour sa part à une «mise en politique de la recherche participative » (p. 45) qui n'aille pas dans le sens d'une exclusion des non-professionnels de la recherche. Si la définition de principes conceptuels et opérationnels "robustes » semble nécessaire à l'instar de ce que rappelle Barré, un équilibre est à trouver afin que l'encadrement des pratiques par des prérequis scientifiques n'empêche pas toute forme de développement originale. Notons enfin que toutes les recherches partenariales ou collaboratives ne revendiquent pas le sceau de la scientificité. Quand bien même le caractère scientifique de certaines peut être mis en doute, bien d'autres vertus peuvent leur être prêtées et, partant, pleinement justifier leur existence. La proposition de solutions à des problèmes sociaux en est une et l'on peut dès lors relayer l'appel formulé par Fontan à la fin de sa contribution : «les acteurs du "système institutionnel dominant" doivent favoriser le développement de sas communicationnel et de médiation afin que les organisations et les institutions de la norme puissent se perméabiliser aux propositions provenant de la "marge". En d'autres mots, il s'agit de rendre plus démocratiques les mécanismes de réflexion et d'apprentissage autour des propositions subversives expérimentées par des acteurs qui se mettent volontairement à la marge des façons attendues d'innover » (p. 252-253). On peut également appeler au développement d'une «culture de la recherche», entendue comme «ce qui, dans un environnement de travail, allume et attise chez les non-chercheurEs, ce goût de la recherche, et, chez les chercheurs, ce goût d'accompagner» (Gélineau, Vinet-Bonin, Gervais, 2009: 309) qui peut ou non, selon les ambitions, s'inscrire dans une démarche revendiquée comme scientifique. Le débat est dans tous les cas à poursuivre en s'interrogeant sur ce qui peut être qualifié de «vraie» recherche» (Cohn, 2008) et sur les conditions et les bénéfices de la promotion d'une «science pure».

Les différentes contributions théoriques et empiriques permettent de s'approprier les enjeux associés à de telles pratiques. Elles ne fournissent pas un guide pratique pour qui voudrait les mettre en œuvre, mais conduiront ceux qui réfléchissent à cette méthodologie à identifier les points de vigilance et à clarifier leur positionnement ou, plus précisément, leur posture et celle qu'ils attendent des différents participants à la recherche. En effet, l'un des enjeux est bien celui-là : qui fait quoi dans une recherche collaborative ou partenariale et comment les différents acteurs se positionnent-ils les uns par rapport aux autres ? Autrement dit, qui participe à la recherche, comment et à quel degré chacun participe-t-il ? Si ce n'est pas le vocable retenu par les coordinatrices 
de l'ouvrage, l'expression « recherche participative» est en effet souvent utilisée pour désigner de telles pratiques et il semble à la lecture des contributions que la question de la participation soit effectivement partout présente en creux. Il s'agit à chaque fois de penser et de pratiquer la recherche en «collaborant», en établissant des "partenariats » avec des «non-chercheurs », au sens où ce ne sont pas des chercheurs professionnels (ou des scientifiques), autrement dit de penser et de pratiquer la recherche en faisant «participer» ces «non-chercheurs». Les questionnements mis en évidence par la sociologie de la participation, et notamment la sociologie de la participation citoyenne, pourraient alors venir éclairer de manière, sinon essentielle, au moins judicieuse les problématiques des recherches partenariales et participatives, ce qui n'est que furtivement le cas dans les contributions de cet ouvrage. Les enjeux de ces pratiques en matière d'émancipation et d'empowerment seraient ainsi davantage discutés et valorisés. Pour autant, les apports de différents types et courants sociologiques sont mis en avant. Certaines contributions y sont explicitement dédiées: Frédéric Blondel donne des éclairages à partir de la sociologie clinique, Catherine Bourgeois et Anne Gillet à partir de la sociologie de l'action organisée, Christine Audoux à partir de la sociologie de la traduction et Maurice Blanc et Josiane Stoessel-Ritz à partir de la sociologie de la transaction sociale. L'ensemble des contributions montre ainsi la pertinence de multiplier et de diversifier les regards pour comprendre cet étrange objet que sont les recherches partenariales et collaboratives.

\section{Bibliographie}

Connwall A., Jewkes R. (1995), «What Is Participatory Research?» Social Science \& Medicine, vol. 1, $\mathrm{n}^{\circ}$ 12, p. 1667-1676.

Deroubaix J.-F., De Conink A. (2013), « Recherche partenariale», in I. CASILLO et al. (dir.), Dictionnaire critique et interdisciplinaire de la participation, GIS Démocratie et Participation. En ligne, consulté le 22 janvier 2018. URL: http://www.participation-et-democratie.fr/fr/node/1358.

Callon M., Lascoumes P., Barthe Y. (2001), Agir dans un monde incertain. Essai sur la démocratie technique, Paris, Seuil.

CoHn J. F. (2008), «Citizen Science: Can Volunteers Do Real Research?», BioScience, vol. $58, n^{\circ} 3$, p. $192-197$.

GELINEAU L., Vinet-Bodin L., GERVAIS M. (2009), "Quand recherche et proximité se conjuguent : réflexions autour de l'émergence d'une culture de recherche dans un centre de santé et de services sociaux», in M. Clement et al., Proximités. Lien, accompagnement et soin, Québec, Presses de l’Université du Québec, p. 303-329.

NAUdier D., Simonet M. (dir.) (2011), Des sociologues sans qualités? Pratiques de recherche et engagements, Paris, La Découverte. 
Rene J.-F., Champagne M., Mongeau S. (2013), "Allier recherche et participation citoyenne: enjeux, défis et conditions de réalisation», Nouvelles pratiques sociales, vol. $25, \mathrm{n}^{\circ} 2$, p. 25-34. 